\title{
Computational model for investigating acoustic hemostasis
}

\author{
Maxim A. Solovchuk, ${ }^{* a}$ and Tony W.H. Sheu ${ }^{a, b}$ \\ ${ }^{a}$ Center of Advanced Study in Theoretical Sciences (CASTS), National Taiwan University \\ ${ }^{b}$ Department of Engineering Science and Ocean Engineering, National Taiwan University \\ E-mail: solovchuk@gmail.com, twhsheu@ntu.edu.tw
}

\begin{abstract}
Acoustic hemostasis is a new field of ultrasound research in which high intensity focused ultrasound (HIFU) is used to induce hemostasis. Although it was experimentally shown that focused ultrasound can be used to seal the bleeding site while leaving the vessel patent and to occlude the blood vessel, physical mechanisms of acoustic hemostasis are not fully understood. Quantitative models are required to understand the interaction between different physical mechanisms involved in acoustic hemostasis. In the current work a mathematical model for the study of acoustic hemostasis is presented. This model comprises the nonlinear Westervelt equation and the bioheat equations in tissue and blood vessels. The nonlinear hemodynamic equations are also coupled with the acoustic and thermal equations with the convected cooling and acoustic streaming effects being taken into account.
\end{abstract}

2013 International Workshop on Computational Science and Engineering,

14-17 October 2013

National Taiwan University, Taipei, Taiwan

${ }^{*}$ Speaker. 


\section{Introduction}

Bleeding is one of the leading causes of deaths after traumatic injuries [1]. About 40-50\% of traumatic civilian and battlefield deaths were caused by hemorrhage in recent years [2]. At the present time there are several methods for the hemorrhage control, including ligation, clamping and repair of the vessel $[3,4]$. Acoustic hemostasis is a new field of ultrasound research in which high intensity focused ultrasound (HIFU) is used to induce hemostasis $[5,6]$. Focused ultrasound has been successfully applied for the treatment of tumors in different areas of the bodies, including the breast, prostate, uterine fibroids and liver [7, 8]. Other promising applications of HIFU include blood vessel occlusion [9, 10, 11], hemostasis of bleeding vessels and organs [5, 6, 12].

Although numerous experimental studies have been performed, the optimal strategy for the acoustic hemostasis is not very clear. The ultrasound frequency, intensity, duration of the treatment, location of focal point differ a lot in different experimental studies. The focus can be fixed at one location (for example, at the center of the wound), it can be moved continuously along the wound or multiple sonications at several points. Many studies have been performed for punctured blood vessel $[1,6,13]$. Even for the same ultrasound parameters the treatment time can differ by an order of magnitude for similar punctures [13]. The difference can be attributed to the difference in the wound shape and guidance of HIFU. Ultrasound beam should be precisely located on the wound. If the location of the focal point was not controlled very well, very big increase in treatment time was found. In order to improve the treatment, fundamental understanding of processes during acoustic hemostasis is necessary. In the present paper, the mathematical model for acoustic hemostasis is presented. Importance of thermal effect and acoustic streaming effect has been investigated.

\section{Mathematical model}

\subsection{Nonlinear acoustic equation}

Acoustic field generated by a HIFU source was modeled using the nonlinear Westervelt equation $[14,15,16,17]$ :

$$
\nabla^{2} p-\frac{1}{c_{0}^{2}} \frac{\partial^{2} p}{\partial t^{2}}+\left[\frac{\delta}{c_{0}^{4}}+\frac{2}{c_{0}^{3}} \sum_{v} \frac{c_{v} \tau_{v}}{1+\tau_{v} \frac{\partial}{\partial t}}\right] \frac{\partial^{3} p}{\partial t^{3}}+\frac{\beta}{\rho_{0} c_{0}^{4}} \frac{\partial^{2} p^{2}}{\partial t^{2}}=0
$$

In the above, $p$ is the sound pressure, $\beta=1+\frac{B}{2 A} \quad$ the coefficient of nonlinearity, and $\delta$ the diffusivity of sound resulting from fluid viscosity and heat conduction, $\tau_{v}$ the relaxation time and $c_{v}$ the small signal sound speed increment for the $v$-th relaxation process. The first two terms describe the linear lossless wave propagating at a small-signal sound speed. The third term denotes the loss resulting from thermal conduction and fluid viscosity, and the fourth term accounts for the relaxation processes. The last term accounts for acoustic nonlinearity which may considerably affect thermal and mechanical changes within the tissue. Equation (2.1) can be further transformed to the coupled system of two partial differential equations given below [14]

$$
\begin{aligned}
& \nabla^{2} p-\frac{1}{c_{0}^{2}} \frac{\partial^{2} p}{\partial t^{2}}+\frac{\delta}{c_{0}^{4}} \frac{\partial^{3} p}{\partial t^{3}}+\frac{\beta}{\rho_{0} c_{0}^{4}} \frac{\partial^{2} p^{2}}{\partial t^{2}}+\sum_{v} P_{v}=0 \\
& \left(1+\tau_{v} \frac{\partial}{\partial t}\right) P_{v}=\frac{2}{c_{0}^{3}} c_{v} \tau_{v} \frac{\partial^{3} p}{\partial t^{3}}
\end{aligned}
$$


In the present paper two relaxation processes $(v=2)$ were considered. Unknown relaxation parameters were calculated by minimizing a mean square error between the linear attenuation law and the relaxation model [14].

For the linear Westervelt equation the intensity is equal to $I_{L}=p^{2} / 2 \rho c_{0}$. For the nonlinear case the total intensity is

$$
I=\sum_{n=1}^{\infty} I_{n}
$$

where $I_{n}$ are the corresponding intensities for the respective harmonics $n f_{0}$. The ultrasound power deposition per unit volume is calculated by

$$
q=\sum_{n=1}^{\infty} 2 \alpha\left(n f_{0}\right) I_{n}
$$

The absorption in tissue shown above obeys the following frequency law:

$$
\alpha=\alpha_{0}\left(\frac{f}{f_{0}}\right)^{\eta}
$$

where $\alpha_{0}=8.1 \mathrm{~Np} / \mathrm{m}, \eta=1.0$ and $f_{0}=1 \mathrm{MHz}$ [18].

\subsection{Energy equation for tissue heating}

Whereas hepatic arteries and portal veins irrigate the liver parenchyma, hepatic veins drain blood out of the liver and can therefore be considered as a heat sink. Tumor cells in perivascular region, as a result, may escape from an externally imposed large heat, leading possibly to a local recurrence. Therefore, the mathematical model appropriate for predicting the temperature in tissues must take the heat conduction, tissue perfusion, convective blood cooling, and heat deposition due to incident wave into account. In the simulation of thermal field the physical domain has been split into the domains for the perfused tissue and the flowing blood.

In a region free of large blood vessels, the diffusion-type Pennes bioheat equation [19] given below will be employed to model the transfer of heat in the perfused tissue region

$$
\rho_{t} c_{t} \frac{\partial T}{\partial t}=k_{t} \nabla^{2} T-w_{b} c_{b}\left(T-T_{\infty}\right)+q
$$

In the above bioheat equation proposed for modeling the time-varying temperature in the tissue domain, $\rho, c, k$ denote the density, specific heat, and thermal conductivity, respectively. The subscripts $t$ and $b$ refer to the tissue and blood domains. The notation $T_{\infty}$ is denoted as the temperature at a remote location. The variable $w_{b}\left(\equiv 0.5 \mathrm{~kg} / \mathrm{m}^{3}-\mathrm{s}\right)$ in Eq. (2.6) is the perfusion rate for the tissue cooling in capillary flows. It is noted that the above bioheat equation for $T$ is coupled with the Westervelt equation (2.1) for the acoustic pressure through a power deposition term $q$ defined in Eq. (2.4).

In the region containing large vessels, within which the blood flow can convect heat, the biologically relevant heat source, which is $q$, and the heat sink, which is $-\rho_{b} c_{b} \underline{\mathbf{u}} \cdot \nabla T$, are added to the conventional diffusion-type heat equation. Application of the resulting energy equation given below avoids a possible high recurrence stemming from the tumor cell survival next to large vessels

$$
\rho_{b} c_{b} \frac{\partial T}{\partial t}=k_{b} \nabla^{2} T-\rho_{b} c_{b} \underline{\mathbf{u}} \cdot \nabla T+q
$$


In the above, $\underline{\mathbf{u}}$ is the blood flow velocity. Owing to the presence of blood flow velocity vector $\underline{\mathbf{u}}$ in the energy equation, we know that a biologically sound model applicable to conduct HIFU simulation should comprise a coupled system of acoustic-thermal-hydrodynamic nonlinear differential equations.

Thermal dose developed by Sapareto and Dewey [20] will be applied to give us a quantitative relationship between the temperature and time for the tissue heating and the extent of cell killing. In focused ultrasound surgery (generally above $50{ }^{\circ} \mathrm{C}$ ), the expression for the thermal dose (TD) can be written as:

$$
T D=\int_{t_{0}}^{t_{\text {final }}} R^{(T-43)} d t \approx \sum_{t_{0}}^{t_{\text {final }}} R^{(T-43)} \Delta t
$$

where $R=2$ for $T>=43^{\circ} \mathrm{C}, R=4$ for $37^{\circ} \mathrm{C}<T<43^{\circ} \mathrm{C}$. The value of TD required for a total necrosis ranges from 25 to $240 \mathrm{~min}$ in biological tissues [20, 21, 22]. According to this relation, thermal dose resulting from heating the tissue to $43^{\circ} \mathrm{C}$ for $240 \mathrm{~min}$ is equivalent to that achieved by heating the same tissue to $56^{\circ} \mathrm{C}$ for one second.

\subsection{Acoustic streaming hydrodynamic equations}

Owing to the inclusion of heat sink, which is shown on the right hand side of Eq. (2.7), the velocity of blood flow plus the velocity resulting from the acoustic streaming due to the applied high-intensity ultrasound must be determined. In this study we consider that the flow in large blood vessels is incompressible and laminar. The vector equation for modeling the blood flow motion, subject to the divergence free equation $\nabla \cdot \underline{\mathbf{u}}=0$, in the presence of acoustic stresses is as follows $[23,24]$

$$
\frac{\partial \underline{\mathbf{u}}}{\partial t}+(\underline{\mathbf{u}} \cdot \nabla) \underline{\mathbf{u}}=\frac{\mu}{\rho} \nabla^{2} \underline{\mathbf{u}}-\frac{1}{\rho} \nabla \mathbf{P}+\frac{1}{\rho} \underline{\mathbf{F}}
$$

In the above, $\mathbf{P}$ is the static pressure, $\mu(=0.0035 \mathrm{~kg} / \mathrm{m} \mathrm{s})$ the shear viscosity of blood flow, and $\rho$ the blood density. In Eq. (2.9), the force vector $\underline{\mathbf{F}}$ acting on the blood fluid due to ultrasound is assumed to act along the acoustic axis $\underline{\mathbf{n}}$. The resulting nonzero component in $\underline{\mathbf{F}}$ takes the following form [25]

$$
\underline{\mathbf{F}} \cdot \underline{\mathbf{n}}=-\frac{1}{c_{0}} \nabla \vec{I}=\frac{q}{c_{0}}
$$

\section{Description of the problem}

The single element HIFU transducer used in this study is spherically focused with an aperture of $12 \mathrm{~cm}$ and a focal length of $12 \mathrm{~cm}$. The parameters used in the current simulation are listed in Table 1.[18]

Table 1: Acoustic and thermal properties for the tissue and blood.

\begin{tabular}{|c|c|c|c|c|c|}
\hline Tissue & $c_{0}\left(\frac{\mathrm{m}}{\mathrm{s}}\right)$ & $\rho\left(\frac{\mathrm{kg}}{\mathrm{m}^{3}}\right)$ & $c\left(\frac{\mathrm{J}}{\mathrm{kgK}}\right)$ & $k\left(\frac{\mathrm{W}}{\mathrm{mK}}\right)$ & $\alpha\left(\frac{\mathrm{Np}}{\mathrm{m}}\right)$ \\
\hline tissue & 1540 & 1055 & 3600 & 0.512 & $8.1 * f$ \\
\hline Blood & 1540 & 1060 & 3770 & 0.53 & $1.5 * f$ \\
\hline
\end{tabular}

In this study, the ultrasound of $1.0 \mathrm{MHz}$ insonation is incident from a location that is exterior of the liver tumor. The initial temperature is equal to $37^{\circ} \mathrm{C}$. The vessel diameter is $3 \mathrm{~mm}$. Initial 


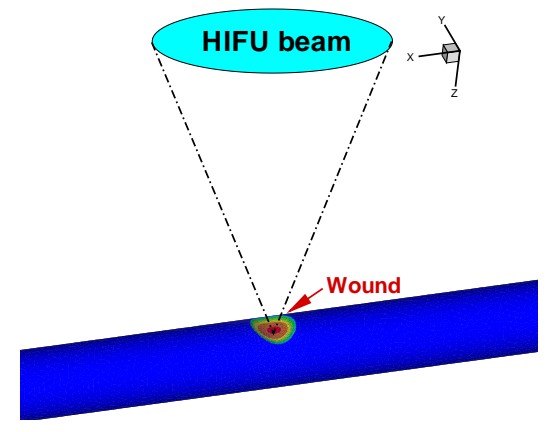

Fig. 1: Geometry of the problem, a small wound with the diameter $d=2 \mathrm{~mm}$ in the blood vessel $(d=3$ $\mathrm{mm})$.

temperature is equal to $37^{0} \mathrm{C}$. The fully developed velocity profile is applied at the inlet of blood vessel, while zero gradient velocity boundary condition on the outlet plane. The pressure at the wound is equal to the tissue pressure. At vessel inlet, the blood flow cross-sectional average velocities are set at $0.016 \mathrm{~m} / \mathrm{s}$ and $0.13 \mathrm{~m} / \mathrm{s}$, that correspond to the velocities in veins and liver arteries with diameter $3 \mathrm{~mm}$ [26]. Two wound shapes, namely, the small circular wound with a diameter 2 $\mathrm{mm}$ and a big wound of $6 \mathrm{~mm}$ in length and $2 \mathrm{~mm}$ in diameter will be investigated (Fig. 1).

In this study, the transducer with the frequency $f_{0}=1.0 \mathrm{MHz}$ is considered. Focal intensity is $2240 \mathrm{~W} / \mathrm{cm}^{2}$, positive and negative pressures are $P_{+}=14 \mathrm{MPa}$ and $P_{-}=6.4 \mathrm{MPa}\left(P_{\text {lin }}=8.5\right.$ $\mathrm{MPa}$ ), respectively, and sonication time is 0.7 second.

A detailed description of the solution procedures can be found in our previous articles [14, 27]. The three-dimensional computational model employed in this study for the prediction of acoustic streaming field was validated by comparing the results with those of Kamakura et al. [23]. The present mathematical model was also validated by comparing our simulated results for the temperature field [24], with and without flow, with the experimental results of Huang et al. [29].

\section{Results and discussion}

\subsection{Thermal effects}

Numerous experimental studies $[6,13,30]$ on acoustic hemostasis disclose the evidence that the thermal effect of focused ultrasound is responsible for hemostasis. The absorbed ultrasound energy in tissue is transformed into the thermal energy during focused therapy and this energy deposition can quickly elevate the tissue temperature. Temperature is increased in excess of $70^{\circ} \mathrm{C}$ in about one second [6]. This temperature increase may seal the bleeding site. The temperature of $70^{\circ} \mathrm{C}$ can be therefore assumed as threshold temperature for the acoustic hemostasis [6].

We first study the temperature distribution in the tissue and in the blood domain during acoustic hemostasis. Focal axis is perpendicular to the blood vessel, focal point is located on the blood vessel wall. The predicted temperature contours at $t=0.7 \mathrm{~s}$ at two cutting planes $x=0.02$ and $y=0$ are depicted in Fig. 2. The temperature inside the blood vessel remains almost unchanged except in the boundary layer closer to blood vessel wall. 


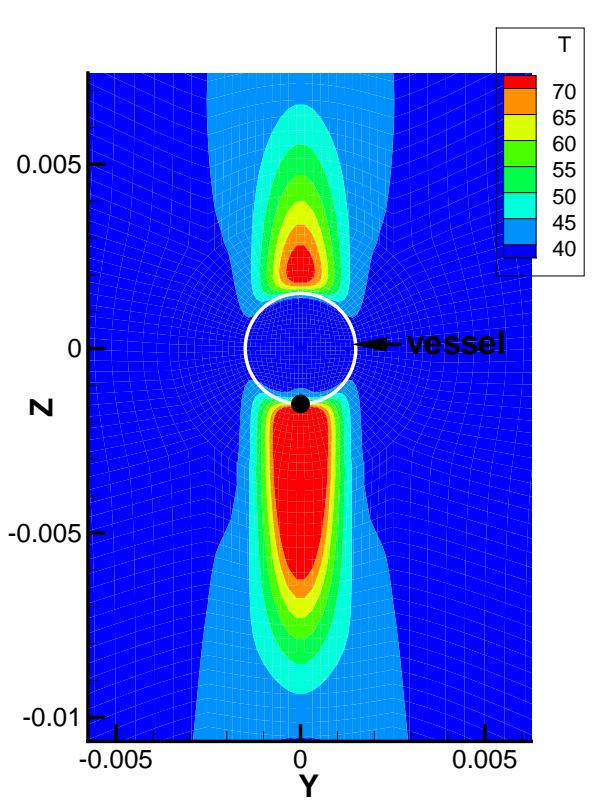

(a)

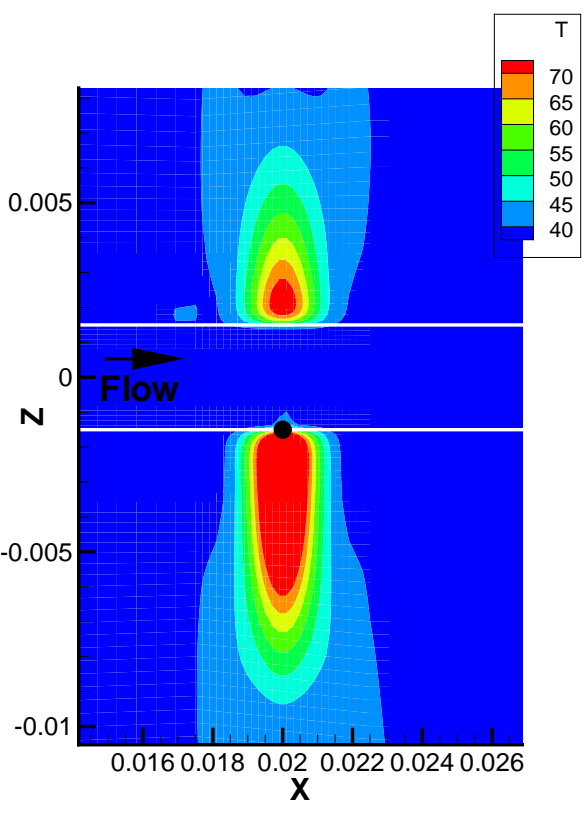

(b)

Fig. 2: The predicted temperature contours at $t=0.7 \mathrm{~s}$ at two cutting planes $x=0.02$ and $y=0$. Focal point (•) is located on the blood vessel wall $(0.02 ; 0 ;-0.0015)$.

The temperature around $70^{\circ} \mathrm{C}$ on the blood vessel wall can be reached at $t=0.7 \mathrm{~s}$. This predicted high temperature shows the theoretical possibility to stop the bleeding. For smaller blood vessels the effects of blood flow cooling and acoustic streaming will be smaller.

In the current subsection we assumed that there is no blood coming out of the wound. In this case the temperature around $70^{\circ} \mathrm{C}$ can be achieved quite rapidly $(t<1 \mathrm{~s})$ and the wound can be sealed in a short time. However, in the real case jet of blood carries away the heat at the bleeding site. As we discussed before, blood also has a lower absorption than a tissue. Therefore if blood is still flowing out of the wound it is quite difficult to seal the wound.

Focused ultrasound can induce an additional mass flow in the liquid. This effect is called acoustic streaming. In the experiments [13] when the the puncture site was exposed to the air, a jet of blood appeared out of the artery after the puncture. A jet of blood was immediately stopped after applying focused ultrasound energy to the bleeding site. So the experiments showed that focused ultrasound can decrease the mass flux out of the wound or even stop bleeding. We are going to investigate the effect of acoustic streaming numerically in the following section.

\subsection{Importance of acoustic streaming}

In the previous studies $[14,27,28]$ it was shown that focused ultrasound can induce acoustic streaming velocities up to $1 \mathrm{~m} / \mathrm{s}$ in the blood vessel and can affect the ultrasound heating. When blood vessel was perpendicular to the acoustic axis, acoustic streaming velocity magnitude was smaller comparing with the parallel blood vessel orientation. 


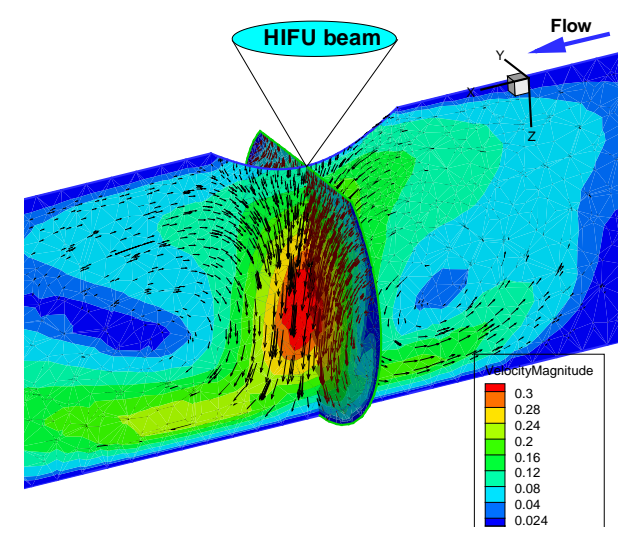

(a)

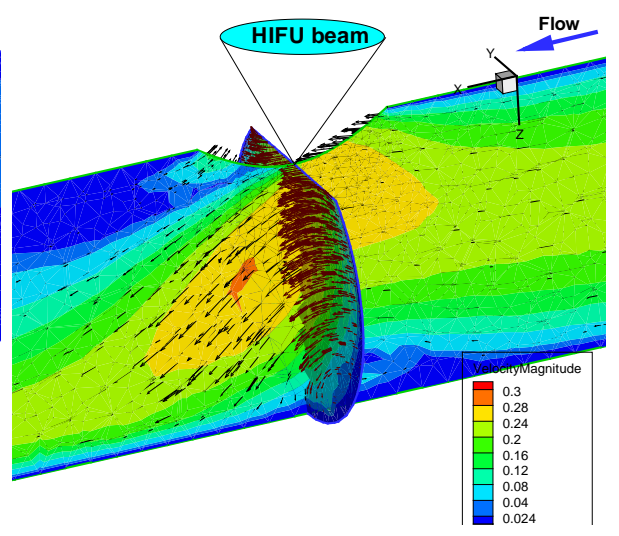

(b)

Fig. 3: Velocity distribution in vein (a) and artery (b) when acoustic streaming effect is taken into account.

When the focus of HIFU transducer is directed towards a bleeding site, local absorption of acoustic energy produces additional momentum to the fluid and results in streaming of the blood away from the focus of transducer. Usually in the acoustic hemostasis experiments blood vessel was located perpendicular to the acoustic axis. However it is not very clear what blood vessel orientation is optimal. For the case of big wound different focal point locations and scanning path method can be chosen in different ways. So we are going to investigate the effect of blood vessel orientation (sonication angle).

Let's consider a hole (wound) on the blood vessel wall. The diameter of the hole is $2 \mathrm{~mm}$, the diameter of the blood vessel is $3 \mathrm{~mm}$, and the maximum velocities are $3.2 \mathrm{~cm} / \mathrm{s}$ (vein) and 26 $\mathrm{cm} / \mathrm{s}$ (artery). Acoustic streaming velocity magnitude is $30 \mathrm{~cm} / \mathrm{s}$, (without acoustic streaming the maximum velocity is $3 \mathrm{~cm} / \mathrm{s}$ ). Acoustic streaming velocity magnitude is one order of magnitude larger than blood flow velocity.

In Figs. 3,4, velocity profiles in vein and artery are presented with and without focused ultrasound. In Tables 2,3, mass fluxes at the inlet and two outlets (outlet and wound) are presented in the vein and in the artery. Mass flux at inlet is equal to $100 \%$. Without AS, $78 \%$ of the total mass flux comes out of the wound. If we switched on the transducer, radiation force will cause acoustic streaming flow to occur. Simulations show that acoustic streaming velocity profile becomes steady within a very short time of $0.12 \mathrm{~s}$.

With acoustic streaming effect being taken into account, the bleeding in the vein can be completely stopped. But there is still a small bleeding out of the artery. In the artery the blood flow out of the wound can be reduced from $45 \%$ to $29 \%$. In order to stop the blood flow out of the wound, a higher power deposition should be considered.

In Fig. 5, the evolution of mass flux out of the wound is presented for different sonication angles in the artery. Focal point is located in the center of the wound. The best sonication angles are from 0 to $90^{0}$. At the angles 0 and $45^{0}$ it's possible to completely stop the flow within $0.1 \mathrm{~s}$. In the experiments, $90^{0}$ sonication is mostly used. However, from simulation point of view it's better to use $45^{\circ}$. This angle allows stopping bleeding even in a large wound.

Sonication angles between 45 and 90 degrees should be considered in order to reduce blood 


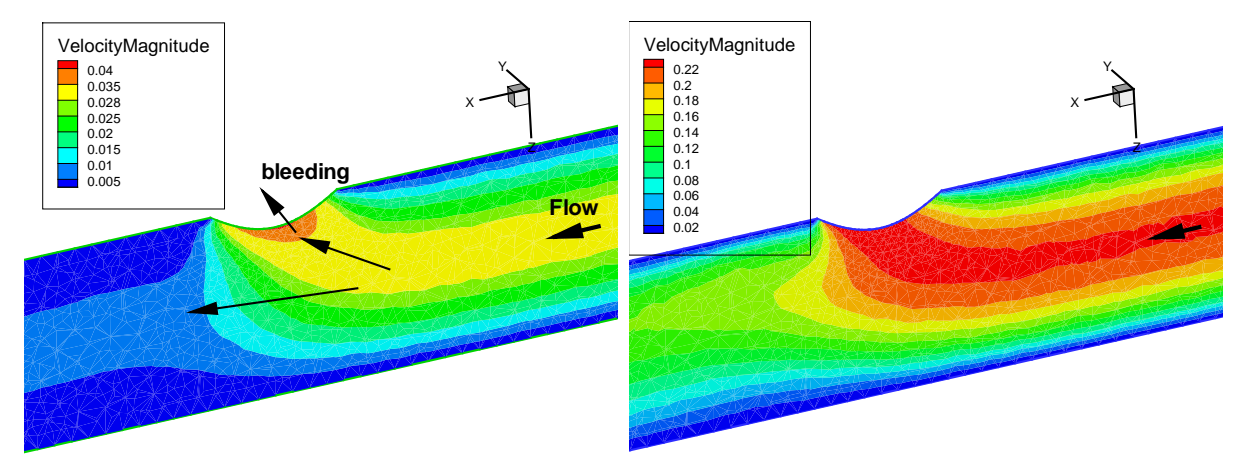

(a)

(b)

Fig. 4: Velocity distribution in vein (a) and artery (b) without acoustic streaming effect.

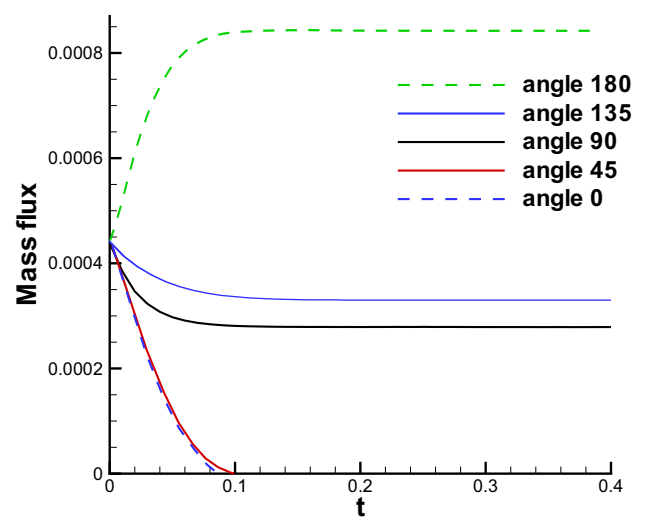

Fig. 5: Evolution of mass flow in the small wound for different sonication angles in the artery.

flow out of the wound. In some cases blood out of the wound can be stopped.

Table 2: Acoustic streaming effect on the mass flux in the small wound in the vein $(u=1.6 \mathrm{~cm} / \mathrm{s})$.

\begin{tabular}{cccc}
\hline Mass flux & Outlet & Wound & Inlet \\
\hline Without AS & $22 \%$ & $78 \%$ & $100 \%$ \\
With AS & $100 \%$ & 0 & $100 \%$ \\
\hline
\end{tabular}

Table 3: Acoustic streaming effect on the mass flux in the small wound in the artery $(u=13 \mathrm{~cm} / \mathrm{s})$.

\begin{tabular}{cccc}
\hline Mass flux & Outlet & Wound & Inlet \\
\hline Without AS & $55 \%$ & $45 \%$ & $100 \%$ \\
With AS & $71 \%$ & $29 \%$ & $100 \%$ \\
\hline
\end{tabular}




\section{Conclusion}

The mathematical model for the investigation of acoustic hemostasis is proposed in the current paper. The model is based on the nonlinear Westervelt equation with the relaxation effect being taken into account and the bioheat equations in the blood vessel and tissue domains. The nonlinear hemodynamic equation is also considered with the acoustic streaming effect being taken into account.

Both thermal and acoustic streaming effects have been investigated in the current paper. Importance of acoustic streaming was studied for different blood vessel orientations. Acoustic streaming velocity magnitude is $32 \mathrm{~cm} / \mathrm{s}$ and is several times larger than velocity in blood vessel. If focused ultrasound beam is applied directly on the bleeding site, the flow out of a wound is considerably reduced due to acoustic streaming. Bleeding can be even completely stopped depending on the blood vessel orientation and focal point location. As a result, the wound can be rapidly sealed. Simulations show that the temperature around $70{ }^{\circ} \mathrm{C}$ can be achieved within a second on the blood vessel wall, if there is no flow out of the wound. The temperature inside blood vessel remains almost unchanged. This confirms the theoretical possibility to seal the bleeding site by means of focused ultrasound. The blood vessel remains patent after the treatment.

The presented mathematical model can be used in the treatment planning for acoustic hemostasis and blood vessel occlusion.

\section{Acknowledgement}

The authors would like to acknowledge the financial support from the Center of Advanced Study in Theoretical Sciences (CASTS).

\section{References}

[1] V. Zderic, A. Keshavarzi, M. L. Noble, M. Paun, S. R. Sharar, L. A. Crum, R. W. Martin, S. Vaezy, Hemorrhage control in arteries using high-intensity focused ultrasound: A survival study, Ultrasonics, 44, 46-53 (2006).

[2] S. Vaezy, V. Zderic, Hemorrhage control using high intensity focused ultrasound, International Journal of Hyperthermia, 23, 203-211 (2007).

[3] O. M. Austin, H. P. Redmond, P. E. Burke, P. A. Grace and H. B. Bouchier, Vascular trauma - $a$ review, J. Am. Coll. Surg., 181, 91-108 (1995).

[4] M. O. Perry, Vascular Trauma, Adv. Surgery, 28, 59-70 (1995).

[5] S. Vaezy, R. Martin, P. Kaczkowski, G. Keilman, B. Goldman, H. Yaziji, S. Carter, M. Caps, L. Crum, Use of high-intensity focused ultrasound to control bleeding, Journal of Vascular Surgery, 29, 533-542 (1999).

[6] S. Vaezy, R. Martin, L. Crum, High intensity focused ultrasound: A method of hemostasis, Echocardiography, 18, 309-315 (2001).

[7] Y. F. Zhou, High intensity focused ultrasound in clinical tumor ablation, World J. Clin. Oncol, 2, 8-27 (2011). 
[8] T. A. Leslie and J. E. Kennedy, High intensity focused ultrasound in the treatment of abdominal and gynaecological diseases, Int. J. Hyperthermia, 23, 173-182 (2007).

[9] K. Hynynen, V. Colucci, A. Chung, F. Jolesz, Noninvasive arterial occlusion using MRI-guided focused ultrasound, Ultrasound in Medicine and Biology, 22, 1071-1077 (1996).

[10] J. Serrone, H. Kocaeli, T. Douglas Mast, M. T. Burgess, M. Zuccarello, The potential applications of high-intensity focused ultrasound (HIFU) in vascular neurosurgery, Journal of Clinical Neuroscience, 19, 214-221 (2012).

[11] P. W. Henderson, G. K. Lewis, N. Shaikh, A. Sohn, A. L. Weinstein, W. L. Olbricht, J. A. Spector, A portable high-intensity focused ultrasound device for noninvasive venous ablation, Journal of Vascular Surgery, 51, 707-711 (2010).

[12] S. Vaezy, R. W. Martin, U. Schmiedl, M. Caps, S. Taylor, K. Beach, S. Carter, P. Kaczkowski, G. Keilman, S. Helton, W. Chandler, P. Mourad, M. Rice, R. Roy, L. Crum, Liver hemostasis using high-intensity focused ultrasound, Ultrasound in Medicine and Biology, 23(9), 1413-1420 (1997).

[13] S. Vaezy, R. Martin, H. Yazij, P. Kaczkowski, G. Keilman, S. Carter, M. Caps, E. Y. Chi, M. Bailey, L. Crum, Hemostasis of punctured blood vessels using high-intensity focused ultrasound, Ultrasound Med Biol., 24, 903-910 (1998).

[14] M. A. Solovchuk, T. W. H. Sheu, M. Thiriet, Simulation of nonlinear Westervelt equation for the investigation of acoustic streaming and nonlinear propagation effects, J. Acoust. Soc. Am., 134(5), 3931-3942 (2013).

[15] M. A. Solovchuk, T. W. H. Sheu, M. Thiriet, Image-based computational model for focused ultrasound ablation of liver tumor, Journal of Computational Surgery 1:4 (2014).

[16] M. F. Hamilton, D. T. Blackstock, Nonlinear Acoustics, Academic Press, Boston, 1998.

[17] Y. Jing, R. O. Cleveland, Modeling the propagation of nonlinear three-dimensional acoustics beams in inhomogeneous media, J. Acoust. Soc. Am. 122, 1352-1364 (2007).

[18] F. A. Duck, Physical Property of Tissues - A comprehensive reference book, Academic, London, 1990.

[19] H. H. Pennes, Analysys of tissue and arterial blood temperature in the resting human forearm, J. Appl. Physiol. 1(2), 93-122 (1948).

[20] S. A. Sapareto, W. C. Dewey, Thermal dose determination in cancer therapy, Int. J. Radiat. Oncol. Biol. Phys. 10(6), 787-800 (1984).

[21] T. W. H. Sheu, M. A. Solovchuk, A. W. J. Chen, M. Thiriet, On an acoustics-thermal-fluid coupling model for the prediction of temperature elevation in liver tumor, Int. J. Heat and Mass Transfer, 54(17-18), 4117-4126(2011).

[22] P. M. Meaney, M. D. Cahill and G. R. ter Haar, The intensity dependence of lesion position shift during focused ultrasound surgery, Ultrasound Med. Biol., 26(3), 441-450 (2000).

[23] T. Kamakura, M. Matsuda, Y. Kumamoto, M. A. Breazeale, Acoustic streaming induced in focused Gaussian beams, J. Acoust. Soc. Am., 97, 2740-2746 (1995).

[24] M. A. Solovchuk, T. W. H. Sheu, W. L. Lin, I. Kuo, and M. Thiriet, Simulation study on acoustic streaming and convective cooling in blood vessels during a high-intensity focused ultrasound thermal ablation, Int. J. Heat and Mass Transfer 55, 1261-1270 (2012). 
[25] W. L. Nyborg, Acoustic Streaming in: M. F. Hamilton, D. T. Blackstock (Eds.), Nonlinear Acoustics, (Academic Press, San Diego, 1998), Ch. 7.

[26] J. W. Hand, Ultrasound hyperthermia and the prediction of heating, in: F. A. Duck, A. C. Baker, H. C. Starritt (Eds.), Ultrasound in Medicine, Institute of Physics Publishing, Bristol, 1998, Ch. 8.

[27] M. A. Solovchuk, T. W. H. Sheu, M. Thiriet, W. L. Lin, On a computational study for investigating acoustic streaming and heating during focused ultrasound ablation of liver tumor, J. of Applied Thermal Engineering, 56(1-2), 62-76 (2013)

[28] M. A. Solovchuk, T. W. H. Sheu, M. Thiriet, Effects of acoustic nonlinearity and blood flow cooling during HIFU treatment, AIP Conf. Proc. 1503, 83-88 (2012).

[29] J. Huang, R. G. Holt, R. O. Cleveland, R. A. Roy, Experimental validation of a tractable medical model for focused ultrasound heating in flow-through tissue phantoms, J. Acoust. Soc. Am., 116(4), 2451-2458 (2004).

[30] S. Vaezy, M. L. Noble, A. Keshavarzi, M. Paun, A. F. Prokop, C. Cornejo, S. Sharar, E. Y. Chi, L. A. Crum, R. W. Martin, Liver Hemostasis with High-Intensity Ultrasound: Repair and Healing, Journal of Ultrasound in Medicine, 23(2), 217-225 (2004).

[31] M. A. Solovchuk, T. W. H. Sheu, M. Thiriet, The effects of acoustic streaming on the temperature distribution during focused ultrasound therapy, AIP Conf. Proc. 1433, 589-592 (2012). 